\title{
CHEMICAL POTENTIAL EVIDENCE FOR PHASE TRANSITIONS IN MAGNETIC AND SUPERCONDUCTING COMPOUNDS AND ALLOYS
}

\author{
M. Matlak ${ }^{a}$ And M. Pietruszka ${ }^{b}$ \\ ${ }^{a}$ Institute of Physics, University of Silesia, Uniwersytecka 4, 40-007 Katowice, Poland \\ ${ }^{b}$ Faculty of Biology and Environmental Protection, University of Silesia \\ Jagiellońska 28, 40-032 Katowice, Poland
}

We announce that all phase transitions (induced by temperature or concentration) including structural ones and transitions between metastable or "exotic" states can be detected by the chemical potential critical behaviour, as well as, from the average occupation numbers of the electronic system (critical electron redistribution).

PACS numbers: $74.70 .-\mathrm{b}, 75.30 . \mathrm{Kz}, 75.40 . \mathrm{Cx}$

We assume that the observable properties of the rare earth based ReX compounds [1] can be described [2] by the following model Hamiltonian:

$$
\bar{H}_{\mathrm{ReX}}=H_{\mathrm{Re}}+H_{\mathrm{X}}+H_{\mathrm{Re}-\mathrm{X}}-\mu N \text {, }
$$

where

$$
\begin{aligned}
& H_{\mathrm{Re}}=-\frac{1}{2} \sum_{i j} J_{i j} S_{i} \cdot S_{j}-\frac{1}{2} g \sum_{i} S_{i}^{z}\left(n_{i \uparrow}^{c}-n_{i \downarrow}^{c}\right) \\
& \quad+\sum_{i j \sigma} t_{i j}^{c} c_{i \sigma}^{+} c_{j \sigma}+\sum_{i j} R_{i j}^{c} c_{i \uparrow}^{+} c_{j \downarrow}^{+} c_{j \downarrow} c_{i \uparrow}, \\
& H_{\mathrm{X}}=\sum_{i^{\prime} j^{\prime} \sigma} t_{i^{\prime} j^{\prime}}^{d} d_{i^{\prime} \sigma}^{+} d_{j^{\prime} \sigma}+\sum_{i^{\prime} j^{\prime}} R_{i^{\prime} j^{\prime}}^{d} d_{i^{\prime} \uparrow}^{+} d_{j^{\prime} \downarrow}^{+} d_{j^{\prime} \downarrow} d_{i^{\prime} \uparrow}, \\
& H_{\mathrm{Re}-\mathrm{X}}=V \sum_{i j^{\prime} \sigma}\left(c_{i \sigma}^{+} d_{j^{\prime} \sigma}+d_{j^{\prime} \sigma}^{+} c_{i \sigma}\right)-\frac{1}{2} \sum_{i j^{\prime}} g_{i j^{\prime}} S_{i}^{z}\left(n_{j^{\prime} \uparrow}^{d}-n_{j^{\prime} \downarrow}^{d}\right)
\end{aligned}
$$

and $N=\sum_{i \sigma} n_{i \sigma}^{c}+\sum_{i^{\prime} \sigma} n_{i^{\prime} \sigma}^{d}$. The lattice site indices $i$ and $j$ numerate the positions of the Re-ions forming the sc lattice. The conduction $d$-electrons belonging to the cluster $\mathrm{X}$ is described by the Hamiltonian, Eq. (2). The indices $i^{\prime}$ and $j^{\prime}$ numerate the positions of the clusters X forming sc lattice (X placed in the centre of each cube). The Hamiltonian (Eq. (3)) stands for the mutual interactions between $c^{-}(\mathrm{Re})$ and $d$-electrons. To determine $\mu$ we use the constraint 

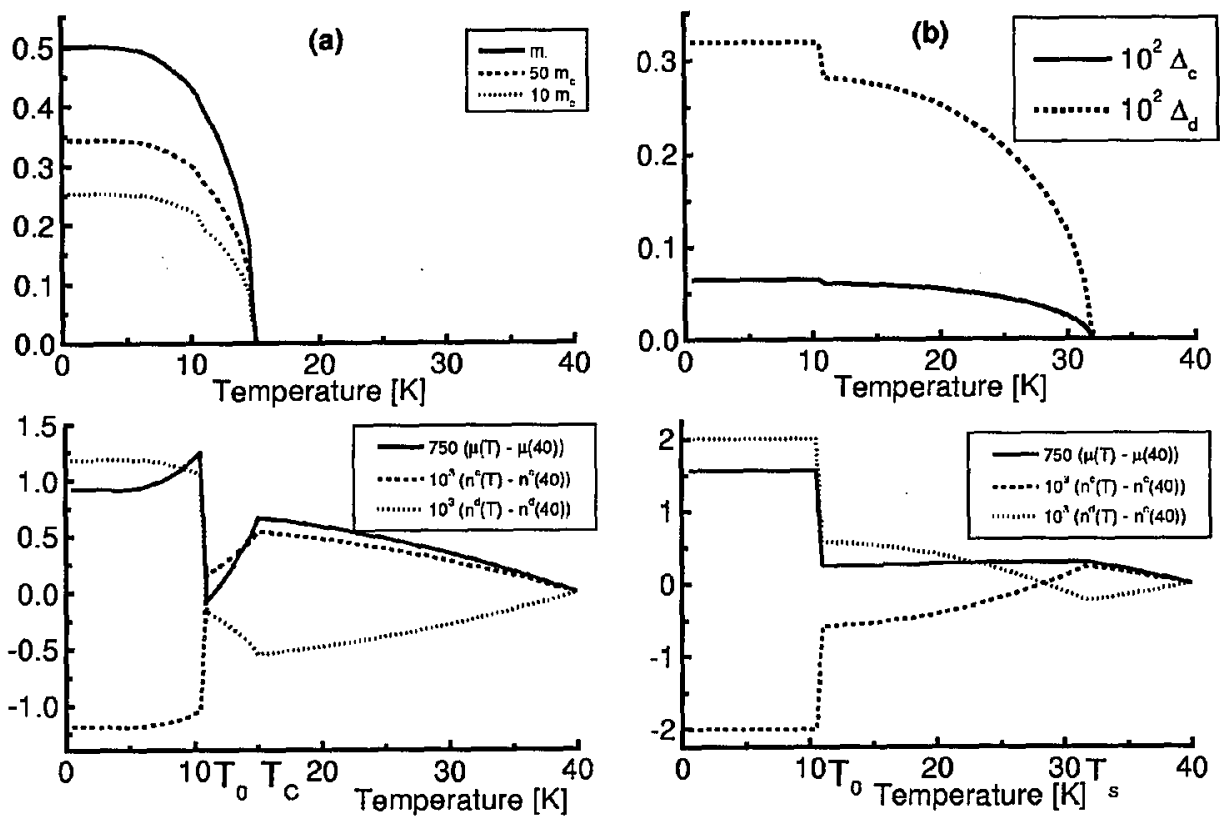

Fig. 1. Plot of $m_{f}$ and $m_{c, d}$ vs. temperature ((a) upper plot). Temperature variation of $\mu[\mathrm{eV}]$ and AON $n^{c, d}$ ((a) lower plot). The model parameters (a): $g=0.05 \mathrm{eV}$, $\bar{g}=\sum_{j^{\prime}} g_{i, j^{\prime}}=0.03 \mathrm{eV}, J_{0}=\sum_{j} J_{i, j}=0.003 \mathrm{eV} ; R_{0}^{c}=R_{0}^{d}=0\left(R_{0}^{c, d}=R_{i, j}^{c, d} ;\right.$ $i, j-$ n.n.). Plot of $\Delta_{c, d}$ ((b) upper plot). Temperature dependence of $\mu[\mathrm{eV}]$ and AON $n^{c, d}\left((\mathrm{~b})\right.$ lower plot). The model parameters (b): $g=\bar{g}=J_{0}=0$ and $R_{0}^{c}=R_{0}^{d}=0.05 \mathrm{eV}$. The common parameters for $(\mathrm{a}, \mathrm{b}): t_{0}^{c}=0, t_{0}^{d}=-0.3 \mathrm{eV}$; hybridization parameter (proportional to $V$, see Ref. [2]) $\varepsilon_{1}=0.1 \mathrm{eV}$; band width ratio $\delta=W^{d} / W=0.1$ $\left(W=W^{c}\right), W_{T \leq T_{0}}=0.48 \mathrm{eV}, W_{T>T_{0}}=0.5 \mathrm{eV}$ and $n=0.1$.

$n^{c}+n^{d}=n\left(n^{c, d}=\sum_{\sigma}\left(\left\langle n_{\sigma}^{c, d}\right\rangle\right)\right)$ and $n$ is the average occupation number (AON) per magnetic ion. We assume $s=1 / 2$ and we consider a single domain case with magnetizations $m_{f}$ and $m_{c, d}$. The superconducting order parameters are denoted as $\Delta_{c}$ and $\Delta_{d}$ [2]. Applying the mean-field approximation, Green's function technique [3], and the true model density of states for sc lattice [4] we calculate the order parameters of the model. We also simulate a structural phase transition (see Ref. [5] for a review) at $T=T_{0}$ introducing two bandwidths $W_{T \leq T_{0}}$ and $W_{T>T_{0}}$.

In Fig. 1a we see that the temperature behaviour of $\mu$ and $A O N$ are strictly correlated with $T_{\mathrm{c}}$ and $T_{0}$. The same correlated behaviour with critical temperatures can be seen in the case of superconducting transition (Fig. 1b). The cases concerning reentrant and non-reentrant phase transitions are depicted in Figs. 2a, b. In the latter case (Fig. 2b) $T_{s_{1}}=0$ and $T_{s_{2}}<T_{\mathrm{c}}$. This exotic situation (similar to Refs. $[6,7])$ is facilitated by the antiparallel alignment of the net magnetizations $\left(m_{c}=-m_{d}\right)$ accompanied by the appearance of two characteristic temperatures $T^{*}$ and $T^{* *}$, connected with metastable states of the system. All critical temperatures, including $T^{*}$ and $T^{* *}$, are reflected in the temperature behaviour of $\mu$ and AON (lower plots in Figs. 2a, b). 

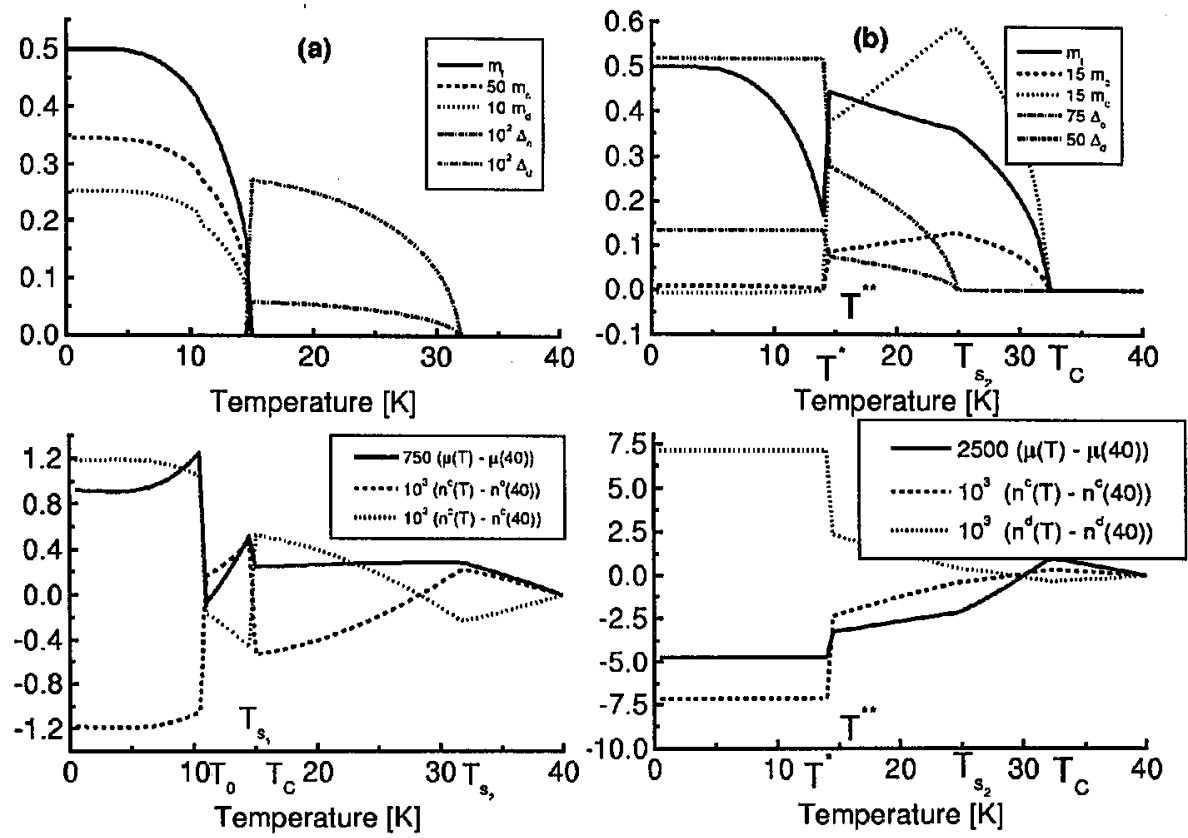

Fig. 2. Plot of $m_{f}, m_{c, d}$ and $\Delta_{c, d}$ in the reentrant case ((a) upper plot). Temperature dependence of $\mu[\mathrm{eV}]$ and $\mathrm{AON} n^{c, d}$ (a) lower plot). The model parameters (a): $g=$ $0.05 \mathrm{eV}, \bar{g}=0.03 \mathrm{eV}, J_{0}=0.003 \mathrm{eV}$ and $R_{0}^{c}=R_{0}^{d}=0.05 \mathrm{eV}$. The other parameters as in Fig. 1. Coexistence of the localized $(4 f)$ ferromagnetism and superconductivity (non-reentrant behaviour) in the case of opposite polarized magnetic moments of $c$ - and $d$-band conduction electrons ((b) upper plot). Temperature dependence of $\mu$ and of AON ((b) lower plot) of $c$ - and $d$-bands. The model parameters (b) are: $W^{c}=W=0.5 \mathrm{eV}$, $J_{0}=0.005 \mathrm{eV}, g=0.06 \mathrm{eV}, \bar{g}=0.04 \mathrm{eV}, \varepsilon_{1}=0.1 \mathrm{eV}, \delta=W^{d} / W=0.1, t_{0}^{c}=0$, $t_{0}^{d}=-0.3 \mathrm{eV}, R_{0}^{c}=R_{0}^{d}=0.075 \mathrm{eV}$ and $n=0.2$.

The Hamiltonian of the $\operatorname{Re}_{1-x}^{(1)} \operatorname{Re}_{x}^{(2)} \mathrm{X}$ alloy in the virtual crystal approximation [8] is $\bar{H}_{\operatorname{Re}_{1-x}^{(1)} \operatorname{Re}_{x}^{(2)} \mathrm{X}}=(1-x) \bar{H}_{\operatorname{Re}^{(1)} \mathrm{X}}+x \bar{H}_{\operatorname{Re}^{(2)} \mathrm{X}}$. To demonstrate the critical behaviour of $\mu$ and $A O N$ in the case of an alloy we first calculate the phase diagram of the system (see the inset in Fig. 3a) which is similar to the one experimentally measured for $\operatorname{Er}_{1-x} \mathrm{Ho}_{x} \mathrm{Rh}_{4} \mathrm{~B}_{4}$ (see e.g. Ref. [1], p. 116). The cross-sections of the phase diagram show the kinks in $\mu$ and AON appearing precisely at all critical concentrations (Fig. 3a, lower plot) and critical temperatures (Fig. 3b, lower plot).

Our observations clearly point the mutual and straightforward relations out between phase transitions and either the critical behaviour of $\mu$ or AON indicating on practical applications of our findings in the experimental condensed matter physics. The theoretical investigations [9] and the experimental measurement of the chemical potential vs. temperature for $\mathrm{YBa}_{2} \mathrm{Cu}_{3} \mathrm{O}_{7-\delta}$ [10] clearly support our results. 
(a)
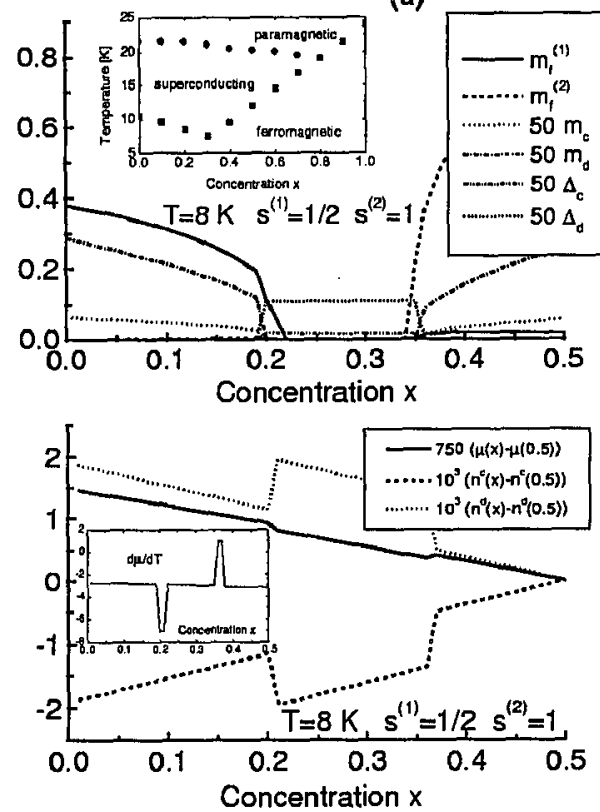

(b)
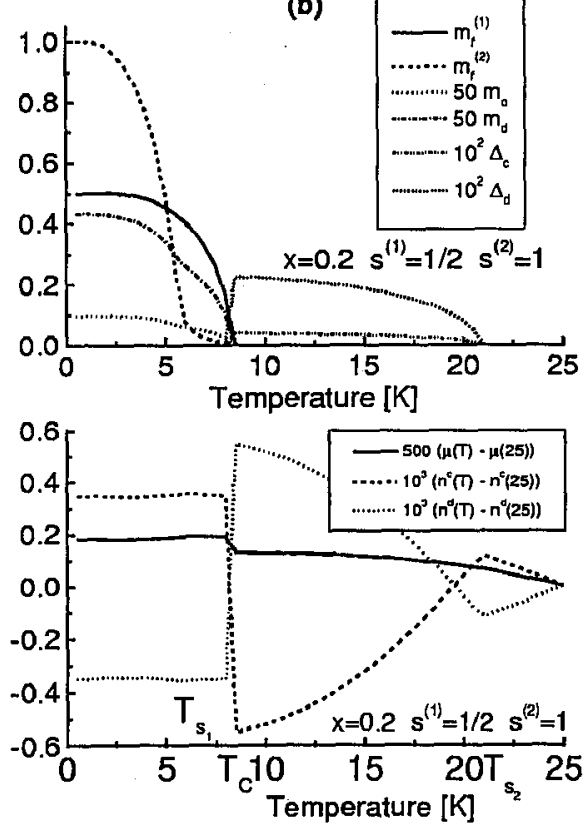

Fig. 3. Plot of $m_{f}^{(\alpha)}, m_{c, d}$ and $\Delta_{c, d}$ vs. concentrations ((a) upper plot) and temperature ((b) upper plot). $\mu[\mathrm{eV}]$ and AON $n^{c, d}$ vs. concentration ((a) lower plot) and temperature ((b) lower plot). The phase diagram of the system (square $-T_{\mathrm{c}}=T_{\mathrm{s}_{1}}$, circle $-T_{\mathrm{s}_{2}}$ ) is given by the inset in (a). The model parameters: $s^{(1)}=1 / 2, s^{(2)}=1, J_{0}^{(1,2)}=0.0035 \mathrm{eV}$, $0.003 \mathrm{eV} ; g^{(1,2)}=0.01 \mathrm{eV}, 0.005 \mathrm{eV} ; \bar{g}^{(1,2)}=0.005 \mathrm{eV}, 0.0045 \mathrm{eV} ; R_{0}^{(1,2) c}=0.04 \mathrm{eV}$, $0.02 \mathrm{eV} ; R_{0}^{d}=0.04 \mathrm{eV}, \varepsilon=0.1 \mathrm{eV}, t_{0}^{(1,2) c}=0,-0.05 \mathrm{eV}, t_{0}^{d}=-0.3 \mathrm{eV}, W^{(1,2) c}=0.5 \mathrm{eV}$, $0.4 \mathrm{eV} ; W^{d}=0.05 \mathrm{eV}$ and $n=0.2$.

\section{References}

[1] M.B. Maple, Physica B 215, 110 (1995).

[2] M. Matlak, M. Pietruszka, Physica C 311, 151 (1999).

[3] A.A. Abrikosov, L.P. Gorkov, I.E. Dzyaloshinski, Quantum Field Theoretical Methods in Statistical Physics, Pergamon Press, Oxford 1965.

[4] R. Jelitto, J. Phys. Chem. Solids 30, 609 (1969).

[5] A.D. Bruce, R.A. Cowley, Structural Phase Transitions, Taylor and Francis Ltd., London 1981.

[6] B.V.B. Sarkissian, A.K. Grover, J. Phys. F 12, L107 (1982).

[7] A. Kolodziejczyk, J. Żukrowski, J. Phys. F 15, L121 (1984).

[8] W. Jones, N.H. March, Theoretical Solid State Physics, Vol. 2, J. Wiley-Interscience, New York 1973, p. 1077.

[9] S. Robaszkiewicz, R. Micnas, K.A. Chao, Phys. Rev. B 20, 3915 (1982).

[10] G. Rietveld, N.Y. Chen, D. van der Marel, Phys. Rev. Lett. 69, 2578 (1992). 